\title{
Ordnung der Gesetze und Gebräuche des Landkrieges
}

\author{
vom 18.Oktober 1907 \\ »Haager Landkriegsordnung«: Auszüge
}

\section{Artikel 1}

Die Gesetze, die Rechte und die Pflichten des Krieges gelten nicht nur für das Heer, sondern auch für die Milizen und Freiwilligen-Korps, wenn sie folgende Bedingungen in sich vereinigen:

1. dass jemand an ihrer Spitze steht, der für seine Untergebenen verantwortlich ist,

2. dass sie ein bestimmtes aus der Ferne erkennbares Abzeichen tragen,

3. dass sie die Waffen offen führen und

4. dass sie bei ihren Unternehmungen die Gesetze und Gebräuche des Krieges beobachten.

\section{Artikel 4}

Die Kriegsgefangenen unterstehen der Gewalt der feindlichen Regierung, aber nicht der Gewalt der Personen oder der Abteilungen, die sie gefangen genommen haben. Sie sollen mit Menschlichkeit behandelt werden.

\section{Artikel 7}

Die Regierung, in deren Gewalt sich die Kriegsgefangenen befinden, hat für ihren Unterhalt zu sorgen. In Ermangelung einer besonderen Verständigung zwischen den Kriegführenden sind die Kriegsgefangenen in Bezug auf Nahrung, Unterkunft und Kleidung auf demselben Fuße zu behandeln wie die Truppen der Regierung, die sie gefangen genommen hat.

\section{Artikel 20}

Nach dem Friedensschlusse sollen die Kriegsgefangenen binnen kürzester Frist in ihre Heimat entlassen werden.

\section{Artikel 22}

Die Kriegführenden haben kein unbeschränktes Recht in der Wahl der Mittel zur Schädigung des Feindes.

\section{Artikel 23}

Abgesehen von den durch Sonderverträge aufgestellten Verboten, ist namentlich untersagt:

a) die Verwendung von Gift oder vergifteten Waffen,

b) die meuchlerische Tötung oder Verwundung von Angehörigen des feindlichen Volkes oder Heeres, c) die Tötung oder Verwundung eines die Waffen streckenden oder wehrlosen Feindes, der sich auf Gnade oder Ungnade ergeben hat,

d) die Erklärung, dass kein Pardon gegeben wird,

e) der Gebrauch von Waffen, Geschossen oder Stoffen, die geeignet sind, unnötig Leiden zu verursachen,

f) der Missbrauch der Parlamentärflagge, der Nationalflagge oder der militärischen Abzeichen oder der Uniform des Feindes sowie der besonderen Abzeichen des Genfer Abkommens,

g) die Zerstörung oder Wegnahme feindlichen Eigentums außer in den Fällen, wo diese Zerstörung oder Wegnahme durch die Erfordernisse des Krieges dringend erheischt wird.

\section{Artikel 25}

Es ist untersagt, unverteidigte Städte, Dörfer, Wohnstätten oder Gebäude, mit welchen Mitteln es auch sei, anzugreifen oder zu beschießen.

\section{Artikel 27}

Bei Belagerungen und Beschießungen sollen alle erforderlichen Vorkehrungen getroffen werden, um die dem Gottesdienste, der Kunst, der Wissenschaft und der Wohltätigkeit gewidmeten Gebäude, die geschichtlichen Denkmäler, die Hospitäler und Sammelplätze für Kranke und Verwundete soviel wie möglich zu schonen, vorausgesetzt, dass sie nicht gleichzeitig zu einem militärischen Zwecke Verwendung finden. Pflicht der Belagerten ist es, diese Gebäude oder Sammelplätze mit deutlichen besonderen Zeichen zu versehen und diese dem Belagerer vorher bekanntzugeben.

\section{Artikel 35}

Die zwischen den abschließenden Parteien vereinbarten Kapitulationen sollen den Forderungen der militärischen Ehre Rechnung tragen. Einmal abgeschlossen, sollen sie von beiden Parteien gewissenhaft beobachtet werden.

\section{Artikel 42}

Ein Gebiet gilt als besetzt, wenn es sich tatsächlich in der Gewalt des feindlichen Heeres befindet. Die Besetzung erstreckt sich nur auf die Gebiete, wo diese Gewalt hergestellt ist und ausgeübt werden kann. 


\section{Artikel 43}

Nachdem die gesetzmäßige Gewalt tatsächlich in die Hände des Besetzenden übergegangen ist, hat dieser alle von ihm abhängenden Vorkehrungen zu treffen, um nach Möglichkeit die öffentliche Ordnung und das öffentliche Leben wiederherzustellen und aufrechtzuerhalten, und zwar, soweit kein zwingendes Hindernis besteht, unter Beachtung der Landesgesetze.

\section{Artikel 46}

Die Ehre und die Rechte der Familie, das Leben der Bürger und das Privateigentum sowie die religiösen Überzeugungen und gottesdienstlichen Handlungen sollen geachtet werden. Das Privateigentum darf nicht eingezogen werden.

\section{Artikel 47}

Die Plünderung ist ausdrücklich untersagt.

\section{Artikel 50}

Keine Strafe in Geld oder anderer Art darf über eine ganze Bevölkerung wegen der Handlungen einzelner verhängt werden, für welche die Bevölkerung nicht als mitverantwortlich angesehen werden kann.

\section{Artikel 56}

Das Eigentum der Gemeinden und der dem Gottesdienste, der Wohltätigkeit, dem Unterrichte, der Kunst und der Wissenschaft gewidmeten Anstalten, auch wenn diese dem Staate gehören, ist als Privateigentum zu behandeln. Jede Beschlagnahme, jede absichtliche Zerstörung oder Beschädigung von derartigen Anlagen, von geschichtlichen Denkmälern oder von Werken der Kunst und Wissenschaft ist untersagt und soll geahndet werden.

\title{
Wissenschaftliches Symposium: Die Europäische Union im 21. Jahrhundert
}

\author{
Bernhard Rinke*
}

Im März diesen Jahres konnte die Europäische Union ein mehr als nur beachtenswertes Jubiläum begehen: Den fünfzigsten Jahrestag der Unterzeichnung der sog. »Römischen Verträge« am 25. März 1957, mit der die bis dahin allein in der Europäischen Gemeinschaft für Kohle und Stahl vereinigten Staaten - Belgien, Frankreich, Italien, Luxemburg, die Niederlande und die Bundesrepublik - die Europäische Wirtschaftsgemeinschaft (EWG) und die Europäische Atomgemeinschaft (EAG) gründeten. Im historischen Rückblick markiert dieses Datum zweifellos einen grundlegenden Meilenstein des europäischen Integrationsprozesses, in dessen Verlauf sich durch Höhen und Tiefen die heutige Europäische Union als Staatenverbund mit weltweit einzigartiger Integrationsdichte entwickelt hat. Am Beginn dieses Prozesses stand - angesichts der Millionen von Toten, Verwundeten und Vertriebenen sowie der ungeheuren Zerstörungen und Gräueltaten des 1. und 2. Weltkriegs - in erster Linie der Wunsch, Krieg als Mittel der Politik ein für allemal zu überwinden. Als innereuropäische Friedensgemeinschaft hat sich die Europäische Gemeinschaft/Europäische Union in den zurückliegenden Jahrzehnten bewährt. Doch an der Schwelle zum 21. Jahrhundert geht es nicht mehr nur um Frieden und Sicherheit in der Europäischen Union, sondern - angesichts vielfältiger alter und neuer sicherheitspolitischer Herausforderungen und Bedrohungen - auch um Frieden und Sicherheit für, mit und durch die Europäische Union. ${ }^{1}$

\footnotetext{
* Dr. Bernhard Rinke, Institut für Friedensforschung und Sicherheitspolitik an der Universität Hamburg (IFSH).

1 Vgl. auch Ehrhart, Hans-Georg et al (Hrsg.): Die Europäische Union im 21. Jahrhundert. Theorie und Praxis europäischer Außen-, Sicherheits- und Friedenspolitik, Wiesbaden 2007.
}

Darin einig waren sich auch die Teilnehmer eines wissenschaftlichen Symposiums unter dem Titel »Die Europäische Union im 21. Jahrhundert«, dass aus Anlass des 60. Geburtstages von Prof. Dr. Dr. h.c. Reinhard Meyers am 27. April 2007 vom Institut für Politikwissenschaft der Westfälischen Wilhelms-Universität Münster veranstaltet wurde. Unter Moderation von Bernhard Rinke diskutierten dabei Dr. Hans-Georg Ehrhart (stellv. Leiter ZEUS), Prof. Dr. Dr. h.c. Wichard Woyke (Professor für Europapolitik, Universität Münster), Prof. Dr. Wilfried Loth (Professor für Neuere und Neueste Geschichte, Universität Duisburg-Essen) und Prof. Dr. Hajo Schmidt (Direktor des Instituts Frieden und Demokratie der FernUniversität Hagen) über die Perspektiven der Europäischen Union im 21. Jahrhundert und ihre friedenspolitische Rolle auf dem Weg zu einer mehr und mehr operativ ausgerichteten Außenund Sicherheitspolitik.

Wichard Woyke fokussierte sich in seinen Ausführungen auf die Zukunft der außen- und sicherheitspolitischen Abschnitte des Europäischen Verfassungsvertrags. Als voraussichtlichen Ausweg aus der gegenwärtigen Verfassungskrise skizzierte er die Umrisse eines »Minivertrags «, der angesichts der großen internationalen Nachfrage nach dem Akteur EU allerdings unbedingt zu einer Stärkung der außen- und sicherheitspolitischen Handlungsfähigkeit der EU führen müsse. Entsprechend plädierte er nachhaltig dafür, das im Verfassungsvertrag vorgesehene Amt eines Europäischen Außenministers zu schaffen. Unter Verweis auf die unterschiedlichen außenpolitischen Traditionen und sicherheitspolitischen Ausrichtungen der EU-Mitgliedstaaten skizzierte er allerdings zugleich das 